\title{
PRODUCT AHP AND ITS PROPERTIES
}

\author{
LIU Qizhi \\ Information school \\ Renmin University of China \\ Beijing P.R.China \\ liuqz@ns.cetin.net.cn
}

Keywords: decision method, multi-criterion decision, analytic hierarchy process, priority

Summary: In this paper we have described Product AHP steps, analyzed its hierarchy structure character, product synthesis character, rank preservation property and alternative set open property, stressed that under a quite general condition the Product Method is the only rank preservation method.

\section{Introduction}

The Analytic Hierarchy Process (AHP) proposed by T.L. Saaty in 1970's has been widely used in corporate planning, portfolio selection, and benefit/cost analysis. It enables us make decision systemically, rationally and quantificationally.

But there are some areas in which the AHP has been criticized from the beginning. One of them is rank reversal [15][16][22]. Many papers discussed how to preserve rank. Product Method is a revision method of AHP with which rank reversal can be solved thoroughly.

The Product Method was proposed in 1988[2] [1]. It has many good properties. In 1995, Liu[3] had proved that under some condition the Product Method is the only rank preservation method furthermore. Although a number of papers [4][5][6][7][8] mentioned the Multiplicative AHP which is similar to Product AHP, but seldom paper discussed this method deeply. In this paper we describe the procedure, foundation theory of Product Method and analyze its properties.

\section{The Product AHP Procedure}

The Product Method can be generalized as four steps: structuring hierarchy, constructing reciprocal matrices, setting priorities of subcriteria, and synthesizing hierarchy results.

\subsection{Structuring hierarchies}

The procedure of structuring hierarchies is the same to traditional AHP, but the concepts are different. The goals, factors named criteria. Between any two criteria A and B, if B has effect on A directly, then it is defined A dominates B; B is the subcriterion of A.

Taking the criteria as points and domination relation as directed edges, there is a directed graph. The AHP use a special directed graph----one root and no circle directed graph. For any node $\boldsymbol{n}$, the longest length of the path from the root to $\boldsymbol{n}$ defined the number of the layer which node $\boldsymbol{n}$ is in. For a node $\boldsymbol{n}$, if it dominates nodes $\boldsymbol{n} \mathbf{1}$ and $\boldsymbol{n} \mathbf{2}, \boldsymbol{n} \mathbf{1}$ and $\boldsymbol{n} \mathbf{2}$ are not in the same level, we can add some virtual nodes and edges revising the graph to satisfy that $\boldsymbol{n} \mathbf{1}$ and $\boldsymbol{n} \boldsymbol{2}$ are in the same level. So not lost generality we can assume that any node $\boldsymbol{n}$ in the graph is just dominated by the same level nodes and the nodes which $\boldsymbol{n}$ dominating are in one level. So the hierarchy structure is like in figure 1: 


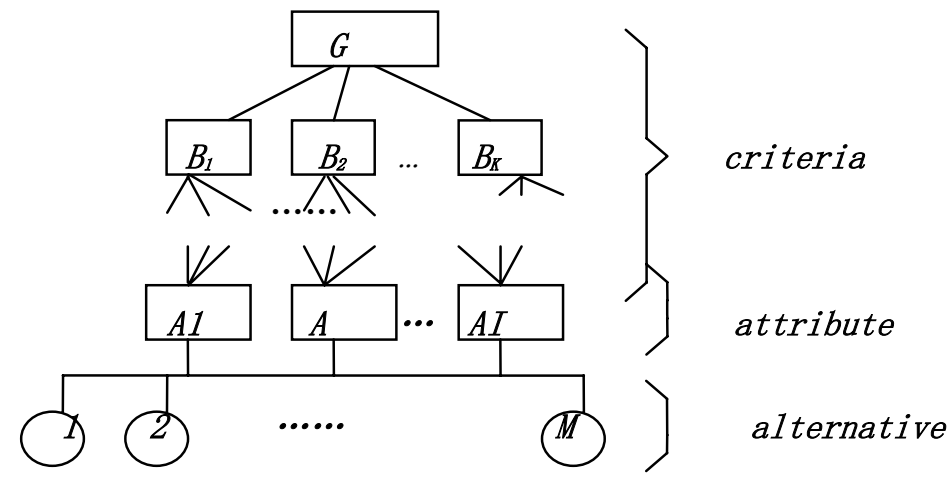

Figure 1

In Product Method the lowest level is distinguished from the criteria, it is named alternative level. The level just above the alternative level is named attribute level.

\subsection{Constructing reciprocal matrices by paired comparisons with ratio scales}

After developing the hierarchy, for every criterion, decision-maker need establish the relationships between its subcriteria by comparing in pairs. When the subcriteria being compared, the 1-9 Scale Table can be used.

1-9 Scale Table

\begin{tabular}{l|l}
\hline Scale & Definition \\
\hline 1 & Equal importance \\
3 & Moderate importance \\
5 & Essential or strong importance \\
7 & Very strong importance \\
9 & Extreme importance \\
$2,4,6,8$ & Intermediate values between the two adjacent judgment \\
Reciprocals & If subcriterion $U i$ has one of the above numbers assigned to it when compared \\
& with subcriterion $U j$, then $U j$ has the reciprocal value when compared with \\
& subcriterion $U i$ \\
\hline
\end{tabular}

Suppose $U_{1}, U_{2}, \ldots, U_{M}$, are subcriteria of criterion $C$, for any two subcriteria $U i$ and $U j$, the decision-maker should judge by ratio scales which one is more important. The result of pairwise comparisons is a positive reciprocal matrix.

Such pairwise comparison is repeated for all the criteria in each level.

The procedure is the same to traditional AHP.

\subsection{Setting priorities of subcriteria dominated by one criterion}

The pairwise comparison matrix is obtained by local observation. The essential priority relationship of the subcriteria can be developed from the matrix. The priority relationship is a positive vector; it is named priority vector (or weighted vector or relative importance vector). Usually the vector is normalized, that is mean the sum of the vector elements is 1 .

There are many methods to calculate the priority vector from the reciprocal matrix, such as Eigenvalue Method, Least Squares Method, and Least Logarithmic Squares Method etc.

The consistency of the pairwise comparison matrix must be tested, if the consistency ratio (C.R.) of C.I. to that from the matrix is significantly small, the matrix passes the consistency test, and otherwise the matrix should be improved.

This step is the same to traditional AHP. 


\subsection{Synthesizing hierarchy results}

\subsubsection{The measurement of criterion importance and the ruler of synthesis}

The criterion importance value is defined recursively as following:

Suppose $A_{1}, A_{2}, \ldots, A_{m}$ are subcriteria of a criterion $B_{I} ;\left(\alpha_{i 1}, \alpha_{i 2}, \ldots, \alpha_{i m}\right)$ is the priority vector of subcriteria $A_{1}, A_{2}, \ldots, A_{m} ; a_{1}, a_{2}, \ldots, a_{m}$ are the importance values of subcriteria $A_{1}, A_{2}, \ldots, A_{m}$ for an alternative respectively. let $b i$ be the importance value of criteria $B i$ for this alternative, then $b i$ is defined as:

$b i=a_{1}^{\alpha_{i 1}} \times a_{2}^{\alpha_{i 2}} \times \ldots . . \times a_{m}^{\alpha m}$

For the criteria in the attribute level, the importance values (attribute values) can be obtained from the reciprocal matrix through step 2.2 and step 2.3, or the attribute values can be obtained by DELPHY method, measurement from alternative directly. For one attribute, it is not necessary to make the elements of all alternatives to be normal (the sun of the elements equal to 1). The different ways can be served simultaneously for different attributes respectively in one decision- making. For convenience and not lost generality, the components of attribute values are restricted to positive numbers, the greater the value is, the better the quality will be. If an attribute does not agree with this property, the value can be transformed to obey the stipulation.

\subsubsection{Bottom up synthesis and top down synthesis}

There are two ways to synthesize, bottom up and top down.

For any alternative, with bottom up synthesis the criteria importance values can be obtained by formula (2.1) recursively from bottom level up to the general criterion.

The general criterion importance value can be written as:

$x_{1}^{\gamma 1} x_{2}^{\gamma 2} \ldots x_{n}^{m}$

The value has two parts, the first is the set of the attribute values, it is a vector $(\boldsymbol{x} 1, \boldsymbol{x} 2, \ldots, \boldsymbol{x n})$. The second is the set of the powers respecting to the attribute importance values. It is also a vector $\left(\gamma_{1}, \gamma_{2}, \ldots\right.$, $\left.\gamma_{n}\right)$.

Top down synthesis calculates the power vector faster. The priority vectors in the same level can be rangged as a priority matrix. The power vector can be obtained by multiplying priority matrices.

The prefer order can be obtained by ranking general criterion importance values of the alternatives.

\section{Example}

If we reduce figure 1 to four levels, general criterion $G$, criteria $\boldsymbol{B}_{1}, \boldsymbol{B}_{2}$ subcriteria, $\boldsymbol{A}_{1}, \boldsymbol{A}_{2}, \boldsymbol{A}_{3}$ and alternative level (figure 2).
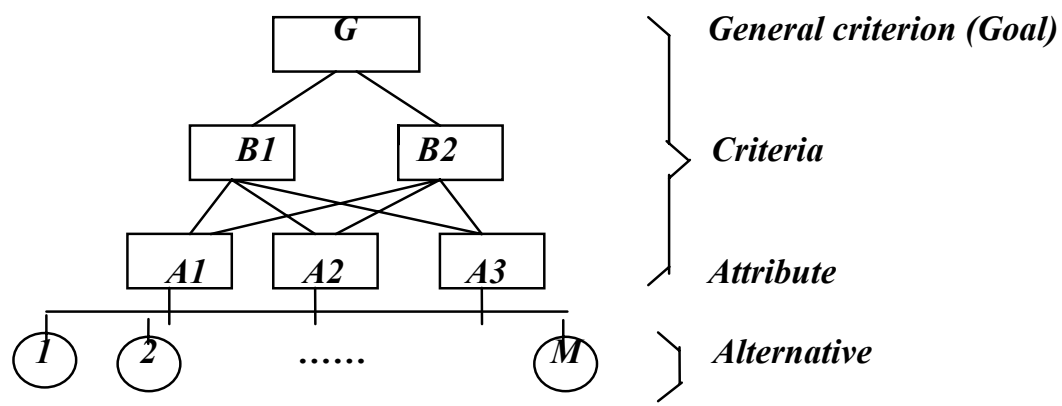

Figure 2

Suppose $\left(\alpha_{11}, \alpha_{12}, \alpha_{13}\right)$ is the priority vector of subcriteria $A_{1}, A_{2}, A_{3}$ dominated by criterion $B_{1} ;\left(\alpha_{21}, \alpha_{22}\right.$, $\left.\alpha_{23}\right)$ is the priority vector of subcriteria $A_{1}, A_{2} A_{3}$ dominated by criterion $B_{2} ;\left(\beta_{1}, \beta_{2}\right)$ is the priority vector of criteria $B_{1}, B_{2}$ dominated by general criterion $G$; $(a 1, a 2, a 3)$ is the importance value vector of subcriteria $A_{l}, A_{2}, A_{3}$ for an alternative $a$. 
If we use Product Method to deal with this example, for the alternative $a$, the importance value of general criterion $G$ is

$$
a_{1}^{c 1} \times a_{2}^{c 2} \times a_{3}^{c 3}
$$

If we use traditional AHP to deal with this example, for the alternative $a$, the importance value of general criterion $G$ is

$$
c_{1} a_{1}+c_{2} a_{2}+c_{3} a_{3}
$$

Priority vector $\left(c_{1}, c_{2}, c_{3}\right)$ is the same, it can be obtained by multiplying priority matrices.

$$
\left(\begin{array}{l}
c_{1} \\
c_{2} \\
c_{3}
\end{array}\right)=\left(\begin{array}{ll}
\alpha_{11} & \alpha_{21} \\
\alpha_{12} & \alpha_{22} \\
\alpha_{13} & \alpha_{23}
\end{array}\right)\left(\begin{array}{l}
\beta_{1} \\
\beta_{2}
\end{array}\right)
$$

The prefer order of alternatives is obtained by ranking general criterion importance values.

\section{The characters and properties of the Product Method}

\subsection{The formula of general criterion importance value}

If we use a vector $\left(x_{1}, x_{2}, \ldots, x_{n}\right)$ to define the attribute values of an alternative, the general criterion importance value of this alternative can be written as a function $\boldsymbol{f}\left(x_{1}, x_{2}, \ldots, x_{n}\right)$. The function $\boldsymbol{f}$ represents the way and preference of decision-maker. In AHP the function $\boldsymbol{f}\left(x_{1}, x_{2} \ldots, x_{n}\right)$ is related with the priority (or weighted) vector $\left(\gamma_{1}, \gamma_{2}, \ldots, \gamma_{n}\right)$. This vector is decided by the hierarchy structure, it is named hierarchy structure vector.

In traditional AHP,

$\boldsymbol{f}\left(x_{1}, x_{2}, \ldots, x_{n}\right)=\gamma_{1} x_{1}+\gamma_{2} x_{2}+\ldots+\gamma_{n} x_{n}$

In Product AHP,

$\boldsymbol{f}\left(x_{1}, x_{2}, \ldots, x_{n}\right)=x_{1}^{\gamma 1} \times x_{2}^{\gamma 2} \times \ldots \times x_{n}^{\gamma_{n}}$

In AHP, any hierarchy can be treated as three levels: general criterion, attribute level and alternative level.

\subsection{Hierarch structure and synthesis characters of Product Method}

When we use Product Method to make decision, there are two characters contrasting with traditional AHP. One is in the hierarchy structure the other is in synthesis.

Although the construction of the hierarchy in Product Method is similar with that in traditional AHP, but the cognition of the hierarchy is difference. In traditional AHP the alternative level is treated as common criterion level and the synthesis ruler (from subcriteria to criterion) must be uniform. In Product Method alternative level is separate from criteria, the criterion synthesis ruler and the ruler of calculating attribute value may be different. When we synthesize from subcriteria to criterion the traditional AHP uses sum of weighted subcriteria, contrast, Product AHP uses product of powered subcriteria.

The product synthesis way has been mentioned in [5][6][7], it is named multiplicative AHP. Because the synthesis ruler for all levels (including alternative level) must be uniform in traditional AHP, therefore the multiplicative AHP cannot extend to Analytic Network Process (ANP). That is a main reason of Saaty refusing multiplicative AHP. Product AHP has overcome this fault. It is easy to extend Product AHP to ANP. We shall discuss the Product ANP in [28].

\subsection{The property of rank preservation}

In AHP, for any two alternatives, suppose the attribute vectors are $\left(\left(x_{1}(1), x_{2}(1), \ldots x_{n}(1)\right)\right.$ and $\left(\left(x_{1}(2), x_{2}(2), \ldots x_{n}(2)\right)\right.$ respectively. 
Property 1. The ratio of two alternative importance values is not changed when any attribute valves multiply same constant in Product Method. That is

$$
\frac{f\left(k_{1} x_{1}(1), \ldots, k_{n} x_{n}(1)\right)}{f\left(k_{1} x_{1}(2), \ldots, k_{n} x_{n}(2)\right)}=\frac{f\left(x_{1}(1), \ldots, x_{n}(1)\right)}{f\left(x_{1}(2), \ldots, x_{n}(2)\right)}
$$

In Product Method if the attribute value is obtained by paired comparisons of alternatives, then the ratio of two alternative importance values does not change, when adding or deleting alternative. If the attribute value is obtained by measurement directly, then the alternative importance values do not change when adding or deleting alternative. So adding or deleting alternative does not affect the alternative priority order. We have proved:

Corollary 1. In Product Method, adding or deleting alternative does not change the alternative priority order

From Corollary 1 we can see that rank reversal in relative measurement [16] will not occur in Product AHP.

From Property 1, the ratio of two alternative importance values does not change when the measurement unit of an attribute is changed. So we have:

Corollary 2. In Product Method, attribute values enlarging or reducing linearly does not change the alternative priority order.

From Corollary 2 we also can see that rank reversal in absolute measurement [1][2] will not occur in Product AHP.

There is a theorem that plays a very important role when we discuss rank reversal and rank preserve in Product AHP.

Theorem. If $\boldsymbol{f}$ is a continue function, not always be 0 , then for all $K_{i}>0, x_{i}(1)>0, x_{i}(2)>0, i=1,2, \cdots, n$

$\frac{f\left(k_{1} x_{1}(1), \ldots, k_{n} x_{n}(1)\right)}{f\left(k_{1} x_{1}(2), \ldots, k_{n} x_{n}(2)\right)}=\frac{f\left(x_{1}(1), \ldots, x_{n}(1)\right)}{f\left(x_{1}(2), \ldots, x_{n}(2)\right)}$

If and only if

$f\left(x_{1}, \ldots, x_{n}\right)=A x_{1}^{\alpha 1} \ldots x_{n}^{\alpha n}$

$A, \alpha_{1}, \alpha_{2}, \ldots, \alpha_{n}$ are all constants.

We leave the proof in next section. Now we clarify what properties the Product Method will have if the theorem is true.

The ratio of two alternative importance values does not change can introduce that the alternatives priority order not changing, but on the contrary it is not true. So the condition of two alternative importance values not changing is stronger than the condition of alternatives priority order not changing. But two alternative importance values not changing is quite a general and convenient condition. From the theorem we see the Product Method is the only method in which the ratio of importance values not changed. So we have:

Property 2. If we strengthen the condition of alternative priority order not changing to be the condition of the ratio of alternative importance values not changing, then the Product Method is the only rank preservation method.

\subsection{The open property of alternative set}

The decision making process with AHP is the ranking or selecting alternative process by decisionmaker's subjective analyses. The attribute vector $\left(x_{1}, x_{2}, \ldots, x_{n}\right)$ is the quantity of attribute, hierarchy structure vector $\left(\gamma_{1}, \gamma_{2}, \ldots, \gamma_{n}\right)$ reflect the preference of decision-maker. In traditional AHP we have accepted that assumption: the formula of general criterion importance value just suit to a certain alternative set. For example, in relative measurement when adding or deleting an alternative the decision 
problem is changed from one to another. Although the situation in absolute measurement is not obvious, but the matter is the same. Because the alternative attribute values are limited to the fixed numbers. Therefore there is a maximum alternative set in absolute measurement. The rank reversal occurs between different alternative sets. Infect the fault is that: on one side accepting the method just suit for the certain decision-making problem, but on the other side contrasting the results between two problems.

In Product Method, the decision function is not changed but the object --alternative set can be opening.

\section{The proof of the theorem}

\section{Proof:}

It is obvious that $(4.2)=>(4.1)$ is true. In the following we prove (4.1) $=>(4.2)$ is true. The proof is carried on Mathematical Induction for dimension number $\boldsymbol{n}$.

First case, when $n=1$ the conclusion is correct.

From the (4.1) is true we have: for all $k_{1}>0, x_{1}(1)>0, x_{1}(2)>0$,

$$
\frac{f\left(k_{1} x_{1}(1)\right)}{f\left(k_{1} x_{1}(2)\right)}=\frac{f\left(x_{1}(1)\right)}{f\left(x_{1}(2)\right)}
$$

Suppose $f(1) \neq 0$, in (5.1). Let $x_{1}(2) \equiv 1, k_{l}=u, x_{l}(1)=v$, then for all $u>0, v>0$ we have: $f(u v)=f(u) f(v) / f(1)$

then if $x>0$ for any natural number $p$ we have:

$f\left(x^{p}\right)=f(1)(f(x) / f(1))^{p}$

Let $x^{p}=z$ then

$f(z)=f(1)\left(f\left(z^{1 / p}\right) / f(1)\right)^{1 / p}$

That is $f\left(z^{1 / p}\right)=f(1)(f(z) / f(1))^{1 / p}$

So for any positive rational number $p / q$, we have:

$f\left(x^{p / q}\right)=f(1)\left(f\left(x^{1 / q}\right) / f(1)\right)^{p}$

$=f(1)(f(x) / f(1))^{p / q}$

Because $\boldsymbol{f}$ is a continue function, so for any two positive real numbers $a, x$, we have:

$f\left(x^{a}\right)=f(1)(f(x) / f(1))^{a}$

Especially let $x=2$, denote $y=2^{a}$

Then $a=\log y / \log 2$ and then

$f(y)=f(1)(f(2) / f(1))^{\log y / \log 2}$

$$
\begin{aligned}
& =f(1)\left((f(2) / f(1))^{\log y}\right)^{1 / \log 2} \\
& =f(1) y^{(\log (f(2) / f(1))) / \log 2} \\
& =A y d
\end{aligned}
$$

In the above formula $A=f(1), d=(\log (f(2) / f(1))) / \log 2$

So we have proved when $n=1,(4.1)=>(4.2)$ is true.

Next we prove if $(4.1)=>(4.2)$ is true for $n$, then $(4.1)=>(4.2)$ is true for $n+1$.

Let the $n$-dimension vectors $\boldsymbol{k}, \boldsymbol{x} \mathbf{1}, \boldsymbol{x} \mathbf{2}$ are defined as:

$$
\begin{aligned}
& \boldsymbol{k}=\left(k_{1}, \cdots, k_{n}\right) \\
& \boldsymbol{x} \mathbf{1}=\left(x_{1}(1), \cdots, x_{n}(1)\right) \\
& \boldsymbol{x} \mathbf{2}=\left(x_{1}(2), \cdots, x_{n}(2)\right)
\end{aligned}
$$

$$
\text { Denote } \boldsymbol{k} \cdot \boldsymbol{x}=\left(k_{1} x_{1}, \cdots, k_{n} x_{n}\right)
$$

$$
\boldsymbol{y} \mathbf{1}=x_{n+1}(1), \boldsymbol{y} \mathbf{2}=x_{n+1}(2), k_{n+1}=a
$$

Then from (4.1) is true for $n+1$ we have:

$$
\frac{f\left(k \bullet x 1, a y_{1}\right)}{f\left(k \bullet x 2, a y_{2}\right)}=\frac{f\left(x 1, y_{1}\right)}{f\left(x 2, y_{2}\right)}
$$

In (5.2), especially let $\mathrm{n}$-dimension vectors $\boldsymbol{k}=(1,1, \cdots, 1), \boldsymbol{x}_{1}=\boldsymbol{x}_{2}=\boldsymbol{x}=\left(x_{1}, x_{2}, \cdots, x_{n}\right)$, then (5.2) can be written as: 
$\frac{f\left(x, a y_{1}\right)}{f\left(x, a y_{2}\right)}=\frac{f\left(x, y_{1}\right)}{f\left(x, y_{2}\right)}$

In (5.3), the function $\boldsymbol{f}(\boldsymbol{x}, y)$ can be deal with as such a function of singular variable of $y$. Because when $n=1$ the conclusion is correct, so we can get:

$\boldsymbol{f}(\boldsymbol{x}, y)=\boldsymbol{f}(\boldsymbol{x}, 1) \cdot y^{(\log (f(x, 2)) f(x, 1))) \log 2}$

Not lost generality, let $f(1, \ldots, 1,1) \neq 0$, because we suppose (4.1) is true for $n+1$, so we have:

$\frac{f(x, 2)}{f(x, 1)}=\frac{f(1, \ldots, 1,2)}{f(1, \ldots, 1,1)}$

And we have supposed (4.1) $=>(4.2)$ is true for $\mathrm{n}$, so we have:

$f(x, 1)=A x_{1}^{\gamma 1} \ldots x_{n}^{m}$

Let $\beta=\left(\log \frac{f(1, \ldots, 1,2)}{f(1, \ldots, 1,1)}\right) / \log 2$

Then (5.4) can be written as:

$f(x, y)=A x_{1}^{\alpha 1} \ldots x_{n}^{\alpha n} \times y^{\beta}$

End.

\section{Conclusion}

In this paper we have described Product AHP procedure, listed its hierarchy structure character, product synthesis character, rank preservation property and alternative set open property, stressed that in a quite general condition the Product Method is the only rank preservation method.

\section{References}

[1]. Liu, Qizhi and Wang, Qin, " Product Method of Analytic Hierarchy Process", Asia-Pacific Journal of Operational Research, 8(1991) 135-145.

[2]. Liu, Qizhi and Wang, Qin, " Product Method of AHP", Proceedings of International Symposium on The Analytic Hierarchy Process, Tianjin, China, 1988, 225-231.

[3]. Liu, Qizhi, Rank Preservation Property of Product AHP, Systems Engineering Journal (Chines), 1995(10) 61-70.

[4]. Saaty, T.L., The Seven Pillars of Analytic Hierarchy Process, Proceedings of The Fifth International Symposium on Analytic Hierarchy Process, Kobe, Japan, 1999, 20-23.

[5]. Saaty, T.L., A Note on Multiplicative Operations in AHP, Proceedings of International Symposium on The Analytic Hierarchy Process, Tianjin, China, 1988,82-86.

[6]. Saaty, T.L., Observations on Multiplicative Composition in The Analytic Hierarchy Process, Proceedings of The 3ed International Symposium on Analytic Hierarchy Process, Washington, DC,USA, 1994,169-174.

[7].Barzilai, J., On The Derivation of AHP Properties, Proceedings of The Fourth International Symposium on The Analytic Hierarchy Process, Burnaby, B.C. Canada, 1996, 244-250.

[8].Ramanathan, R., Group Decision Making Using Multiplicative AHP, Proceedings of The Fourth International Symposium on The Analytic Hierarchy Process, Burnaby, B.C. Canada, 1996,262-272.

[9]. Saaty, T.L., "Concepts, Theory and Techniques", Decision Science, 18(1987) 157-177.

[10]. Saaty, T.L. and Xu Shubo., "Recent Developments in the Analytic Hierarchy Process", Proceedings of International Symposium on The Analytic Hierarchy Process, Tianjin, China, 1988, pp62-79.

[11].Zhao Huanchen, Xu Shubo and He Jinsheng, The Method of AHP - a Simple New Decision Method,Science Press (Chines), Beijing, 1986.

[12].Xu,Shubo, The Principle of AHP, Tianjin University Press (Chines), Tianjin, 1988.

[13].Wang, Lianfen, Xu,Shubo, The Introduction to AHP (Chines), China Renmin University Press, Beijing, 1990. 
[14]. Saaty, T.L. and Vargas, L.G. "The Legitimacy of Rank Reversal", OMEGA, 15(1984) 513-516.

[15].Schoner, B.S. and Wedley, W.C., "Ambiguous Criteria Weights in AHP - Consequence and Solutions", Decision Science, 20(1989) 462-475.

[16].Belton, V. and Gear, A.E., "On a short-coming of Saaty's Method of Analytic Hierarchies", OMEGA, 11(1983)228-230.

[17]. Belton, V. and Gear, A.E., "The Legitimacy of Rank Reversal- a Comment", OMEGA, 13(1985) 143-144.

[18]. Waston, S.R and Freeling A.N.S., "Assessin Attribute Weights", OMEGA, 10(1982) 582-583.

[19]. Saaty, T.L., Vargas, L.G. and Wendell, R.E., "Assessing Attribute Weights by Ratios", OMEGA, 11(1983) 9-12.

[20]. Waston, S.R. and Freeling, A.N.S., "Comment on: Assessing Attribute Weights", OMEGA, 11(1983) 13.

[21]. Vargas, L.G., "A Rejoinder", OMEGA, 13(1985) 249.

[22]. Harker, P.T. and Vargas, L.G., "The Theory of Ratio Estimation: Saaty's Analytic Hierarchy Process", Management Science, 33(1987)1383-1403.

[23]. Saaty, T.L., "Rank and The Controversy About the Axioms of Utility Theory -- A Comparison of AHP and MAUT", Proceedings of 2nd International Symposium on The Analytic Hierarchy Process, Pittsburgh, USA, 1991, 87-112.

[24].Zhao, Wei and Jiang, Bo, Recent Development of AHP, Mathematics In Practice And Theory (Chines) 3(1992)63-70.

[25]. Wang, Lianfen and Zhu, Fengtao, A Summary of Rank Reversal In AHP, Systems Engineering---Theory \& Practice (Chines), 1994(14) 1-6.

[26].Saaty,T.L. Fundamentals of Decision Making and Priority Theory with The Analytic Hierarchy Process, RWS Publication Pittsburgh 1994.

[27].Saaty, T .L and L G Vargas, Decision Making, RWS Publication, Pittsburgh 1994.

[28].Liu, Qizhi, " Product Method of Analytic Network Process",(to appear). 